\title{
The relationship between FDG PET/CT-defined metabolic parameters and the histopathological subtype of oesophageal carcinomas
}

\author{
Ulku Korkmaz ${ }^{1 B, D, E, F}$, Muhammet Bekir Hacioglü2B,D,F, Osman Kostek ${ }^{2 A, C, D, F}$, Necdet Sut ${ }^{3 C}$, Hilmi Kodaz ${ }^{4}$, \\ Bulent Erdogan ${ }^{2 A}$, Funda Ustun ${ }^{1 B, F}$, Mert Saynak ${ }^{5 A, B}$, Ebru Tastekin ${ }^{6 B}$, Irfan Cicin ${ }^{2 A, B}$, Gulay Durmus-Altun ${ }^{1 A, D, E}$
}

'Department of Nuclear Medicine, Faculty of Medicine, Trakya University, Edirne, Turkey 2Department of Medical Oncology, Faculty of Medicine, Trakya University, Edirne, Turkey ${ }^{3}$ Department of Statistics, Faculty of Medicine, Trakya University, Edirne, Turkey ${ }^{4}$ Department of Medical Oncology, Acıbadem Eskişehir Hospital, Eskişehir, Turkey ${ }^{5}$ Department of Radiation Oncology, Faculty of Medicine, Trakya University, Edirne, Turkey ${ }^{6}$ Department of Pathology, Faculty of Medicine, Trakya University, Edirne, Turkey

\section{Abstract}

Purpose: $18 \mathrm{~F}$-fluorodeoxyglucose $\left({ }^{18} \mathrm{~F}-\mathrm{FDG}\right)$ positron emission tomography (PET) combined with computed tomography (CT) scan is accepted as a standard tool in the staging of oesophageal cancer (OC). Histological subtype of tumour is known to be a major determinant of prognosis and metabolic behaviour. In this study, we aimed to evaluate the effect of histological subtypes of OC on standard uptake value ( $\mathrm{SUV}_{\max }$ ), metabolic tumour volume (MTV), and total lesion glycolysis (TLG) obtained by PET/CT, and also to compare this effect with prognosis.

Material and methods: Images and clinical course data of 57 patients who were diagnosed with EC and treated in our hospital between 2009 and 2016 were evaluated in a retrospective manner. PET/CT images were re-analysed in terms of metabolic parameters, and observations were compared with histological subtypes.

Results: No significant difference was observed between histological subtypes with $S_{U V} V_{\text {max }}$ overall survival (OS), or progression-free survival (PFS). Thus, MTV was observed to be related with histological subtype; MTV values of adenocancer patients were significantly higher than those of squamous cell cancer patients.

Conclusions: Metabolic tumour volume was related with histological subtype of OC, but clinical staging, TLG, and $\mathrm{SUV}_{\max }$ values were not related with histological subtype, which may suggest the use of MTV as a routine parameter for OC and inclusion of MTV observations in prognostic scoring.

Key words: oesophageal cancer, PET/CT, squamous cell cancer, $\mathrm{SUV}_{\max }, \mathrm{MTV}$, TLG.

\section{Introduction}

Oesophageal carcinoma (OC) is the eighth most common cancer in the world and the seventh leading cause of cancer-related mortality because of its extremely aggressive nature and poor survival rate [1]. Oesophageal squamous cell carcinoma (OSCC) is the predominant histological subtype in the world. However, oesophageal adenocarci- noma (OAC) subtype is more frequent in Western Europe, while in eastern countries the most common subtype is reported as OSCC [2]. Managing OC is difficult, and the response to treatment is generally unsatisfactory. Surgery is the mainstay of treatment for localised OC, with chemoradiotherapy (CRT) being an alternative treatment [3].

Most studies have demonstrated the value of positron emission tomography (PET)/computed tomography (CT)

Correspondence address:

Ülkü Korkmaz, MD, Department of Nuclear Medicine, Faculty of Medicine, Trakya University, 22030, Edirne, Turkey, phone: + 905327993301,

e-mail: korkmaz.ulku@gmail.com

Authors' contribution:

A Study design · B Data collection · C Statistical analysis · D Data interpretation - E Manuscript preparation · F Literature search · G Funds collection 
within the standard of care for the diagnosis, staging and restaging, planning of treatment, and evaluation of response in OC [4-6].

Since the histological subtype determines the clinical behaviour, recent studies have supported the use of volumetric parameters, such as metabolic tumour volume (MTV) and FDG total lesion glycolysis (TLG), which have also proven to be beneficial as prognostic factors in OC. Theoretically, MTV reflects the metabolically active part of tumour burden. Also, it has been defined as the most accurate predictor of outcome. The current data on this topic are results of highly heterogeneous histological subgroups.

The primary aim of this study was to investigate the effects of MTV, TLG, standardised uptake value $\left(\mathrm{SUV}_{\max }\right)$ and histologic subtypes (adenocancer and SCC) of OC on clinical outcomes. The secondary aim of the study is to evaluate a relation between histological subtypes and PET/CT parameters.

\section{Material and methods}

\section{Patients population}

We retrospectively reviewed the records of patients diagnosed and treated with OC between 2009 and 2016 in Trakya University Medical Oncology Department. A total of 18,216 patients were analysed, and 57 patients (28 with OSCC and 29 patients with OAC) who fulfilled the criteria, with evaluation of PET/CT imaging in our local centre and followed by our oncology clinic, were included in the study. A detailed patient selection process is presented in Figure 1. The cases were divided into three clinical stages

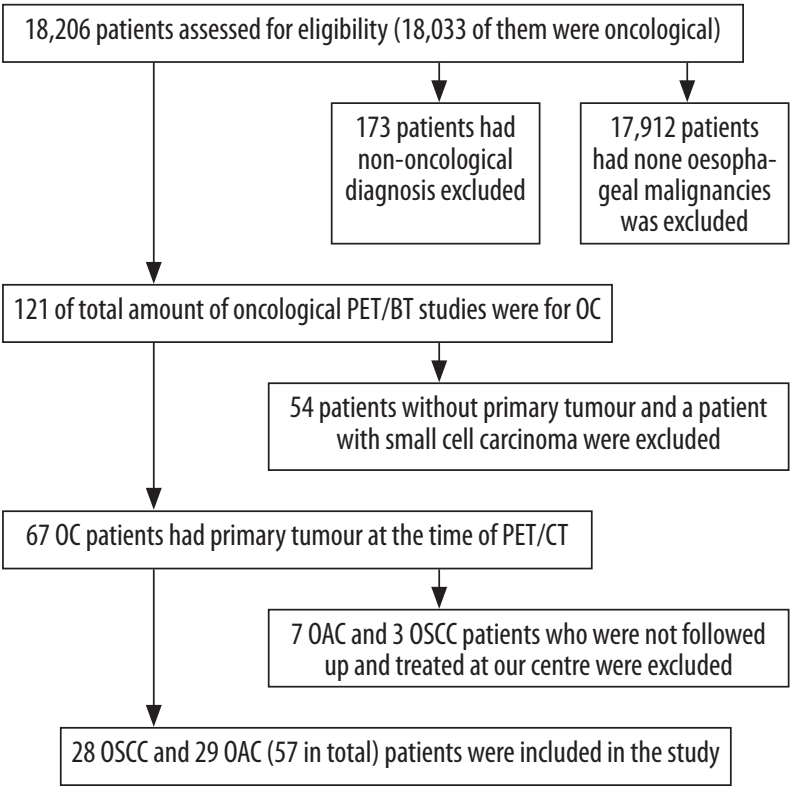

PET - positron emission tomography, $\mathrm{BT}$ - , $\mathrm{OC}$ - oesophageal carcinoma, $\mathrm{OAC}$ - oesophageal adenocarcinoma, $\mathrm{OSCC}$ - oesophageal squamous cell carcinoma

Figure 1. Flow chart of patient selection process as early, locally advanced, and metastatic disease. Because of the low number of patients in the treatment subgroups (surgery alone $[n=7]$, surgery + adjuvant chemotherapy $[n=11]$, surgery + neoadjuvant chemotherapy $[n=14]$, curative CRT [ $n=14]$, palliative therapy [ $n=15]$ ) only histological subgroups were used for further statistical analysis. PET/CT was performed before treatment in all of the patients.

Consent was obtained from all participants included in the study, so that their data could be used for scientific purposes. This study was approved by the local Ethics Committee.

\section{Positron emission tomography/computed tomography images}

PET/CT imaging was performed with a combined wholebody PET/CT system (Discovery STE; GE Medical Systems, Milwaukee, WI). All patients were fasted for at least six hours (blood glucose level $<200 \mathrm{mg} / \mathrm{dl}$ ) with pre-imaging oral contrast hydration, beginning about 60 minutes after intravenous injection of 370-555 MBq (10-15 mCi) FDG. After scanning with low-dose CT for attenuation correction and to definite anatomic localisation without contrast enhancement, patients were analysed using a three-dimensional mode with a three-minute intake and six or seven bed positions from the top of the skull to the upper thigh. After decay and scatter correcting, PET data were reconstructed with attenuation correction and redirected to axial, sagittal, and coronal sections for threedimensional reconstruction.

\section{Image analysis}

PET/CT images were reviewed individually by three authors (UK, FU, GDA), who were board-certified nuclear medicine specialists with over five years of experience in $\mathrm{PET} / \mathrm{CT}$ reading. The lesions with different interpretations among the experts were re-evaluated together and a consensus decision was obtained. Finally, all three experts agreed on all of the lesions evaluated. Focal FDG uptake area with higher density than the neighbouring tissue that did not correspond to physiological uptake sites was accepted as positive for tumour. A spherical volumetric region of interest was drawn semi-automatically over the primary tumour site in the oesophagus and the area of the suspected metastasis. Metabolic tumour volume, $\mathrm{SUV}_{\max }$, and TLG were calculated and recorded automatically for each lesion using PET VCAR software (Advanced Workstation 4.4; GE Medical Systems). Metabolic tumour volume was defined from the region enclosed by a $42 \%$ isocontour threshold around the maximum uptake voxel of the primary tumour. The VOI border was corrected manually if the volume extended beyond the borders of the primary lesion on checking the sagittal and coronal images and the counterpart CT images. All patients had 

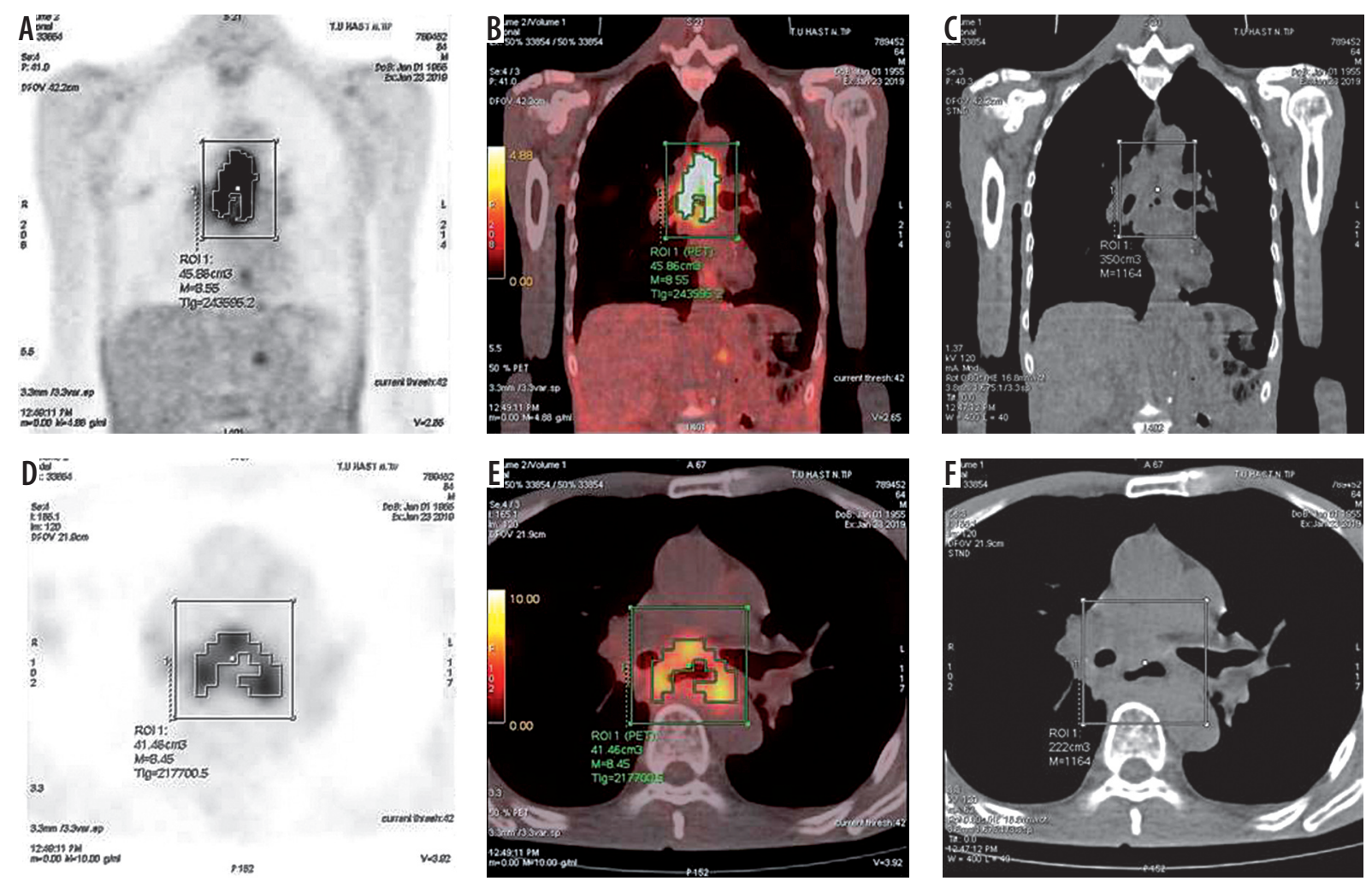

Figure 2. Standardised uptake value, metabolic tumour volume, and total lesion glycolysis calculation of primary tumour of an oesophageal adenocarcinoma patient

normal body mass index, and standardised uptake values (SUVs) were calculated as follows: SUV $=$ radioactivity in ROI $(\mathrm{Bq} / \mathrm{ml}) \times$ lean body mass $(\mathrm{kg}) /$ injected radioactivity $(\mathrm{Bq})$. TLG was calculated using the following formula: $\mathrm{TLG}=\mathrm{SUV}_{\text {mean }} \times \mathrm{MTV}\left(\mathrm{cm}^{3}\right)$ described as by the Usmanij et al. [7]. Figure 2 shows the $S \mathrm{SV}_{\text {max }}, \mathrm{MTV}$, and TLG calculation of primary tumour and metastasis of an EAC from to the same patient.

\section{Statistical analysis}

Categorical variables were compared by chi-square test. The Mann-Whitney U test was used for comparison of demographic and metabolic parameters by histopathological subtypes (EAC vs. ESCC) and mortality (alive vs. dead). Overall and progression-free survival time by histological types were analysed by Kaplan-Meier Survival analysis, and then the Log-rank test was used for comparison of the types.

Diagnostic accuracies of the MTV, TLG, SUV max $_{\text {ax }}$ on histological subtypes and prognosis, and cut-off thresholds were analysed by using the receiver operating characteristics (ROC) curve.

Statistical analyses were performed by using the SPSS 20.0 version (IBM Corp. Released 2011. IBM SPSS Statistics for Windows, Version 20.0. Armonk, NY: IBM Corp.). A $p$ value $<0.05$ was considered as statistically significant.

\section{Patient characteristics}

Of a total of 57 patients, 29 with (50.9\%) OAC and 28 with $(49.1 \%)$ OSCC were included in the study. Primary tumours were predominantly located in the distal oesophagus $(78.9 \%, n=45)$. The mean age of the patients with proximal or distal location differed. Patients with proximal OC were significantly younger (proximal oesophagus: mean 45 , distal oesophagus mean 61 , $p=0.004)$. Although tumour localisation was not related with histology ( $p=0.002)$, OACs were observed to be proximally localised (only one OAC was distally localised). Thirteen patients were observed to have early stage disease $(24.5 \%)$, while 24 had advanced $(45.3 \%)$, and 16 had metastatic disease (30.2\%). The general features of the patients are summarised in Table 1.

\section{Results}

\section{Treatment}

Seven patients received surgery alone (13.2\%), while 11 had surgery with adjuvant chemotherapy (20.7\%), 14 had surgery and neoadjuvant chemotherapy (11.3\%), 14 had curative CRT (26.4\%), and 15 had palliative therapy alone (28.4\%). Because of the low number of patients in the treatment subgroups, no further evaluation was made for these. 
Table 1. Demographic and clinical characteristics of the patient cohort

\begin{tabular}{|c|c|c|}
\hline \multirow[t]{2}{*}{ Parameter } & \multicolumn{2}{|c|}{ Patients } \\
\hline & $n$ & $\%$ \\
\hline \multicolumn{3}{|l|}{ Age, years } \\
\hline Median & 60 & \\
\hline Range & $36-84$ & \\
\hline \multicolumn{3}{|l|}{ Gender } \\
\hline Female & 17 & 29.8 \\
\hline Male & 40 & 70.2 \\
\hline \multicolumn{3}{|l|}{ Histology } \\
\hline Adenocarcinoma & 29 & 50.9 \\
\hline Squamous cell carcinoma & 28 & 49.1 \\
\hline \multicolumn{3}{|l|}{ Tumour location } \\
\hline Proximal oesophagus & 12 & 21.1 \\
\hline Distal oesophagus & 45 & 78.9 \\
\hline \multicolumn{3}{|l|}{ Clinical stage } \\
\hline Early stage & 11 & 19.3 \\
\hline Locally advanced stage & 30 & 52.6 \\
\hline Metastatic stage & 16 & 28.1 \\
\hline \multicolumn{3}{|l|}{ Type of treatment } \\
\hline Surgery alone & 5 & 8.8 \\
\hline Surgery + adjuvant chemotherapy & 10 & 17.5 \\
\hline Surgery + neoadjuvant chemotherapy & 6 & 10.5 \\
\hline Curative chemoradiotherapy & 21 & 36,9 \\
\hline Palliative therapy & 15 & 26.3 \\
\hline
\end{tabular}

Table 2. Descriptive data of the distribution of distant metastases according to organ, histological subgroup, and primary tumour location

\begin{tabular}{|l|c|c|c|c|c|}
\hline Organ & EAC & ESCC & $\begin{array}{c}\text { Proximal } \\
\text { tumour }\end{array}$ & $\begin{array}{c}\text { Distal } \\
\text { tumour }\end{array}$ & Total \\
\hline Lung & 6 & 4 & 3 & 7 & 10 \\
\hline Adrenal & 7 & 1 & - & 8 & 8 \\
\hline Liver & 4 & 1 & - & 5 & 8 \\
\hline Bone & 4 & 3 & 1 & 6 & 7 \\
\hline Brain & 1 & - & - & 1 & 1 \\
\hline PVT & 1 & - & - & 1 & 1 \\
\hline
\end{tabular}

OAC - oesophageal adenocancer, OSCC - oesophageal squamous cell cancer, PVT - porta vein thrombosis

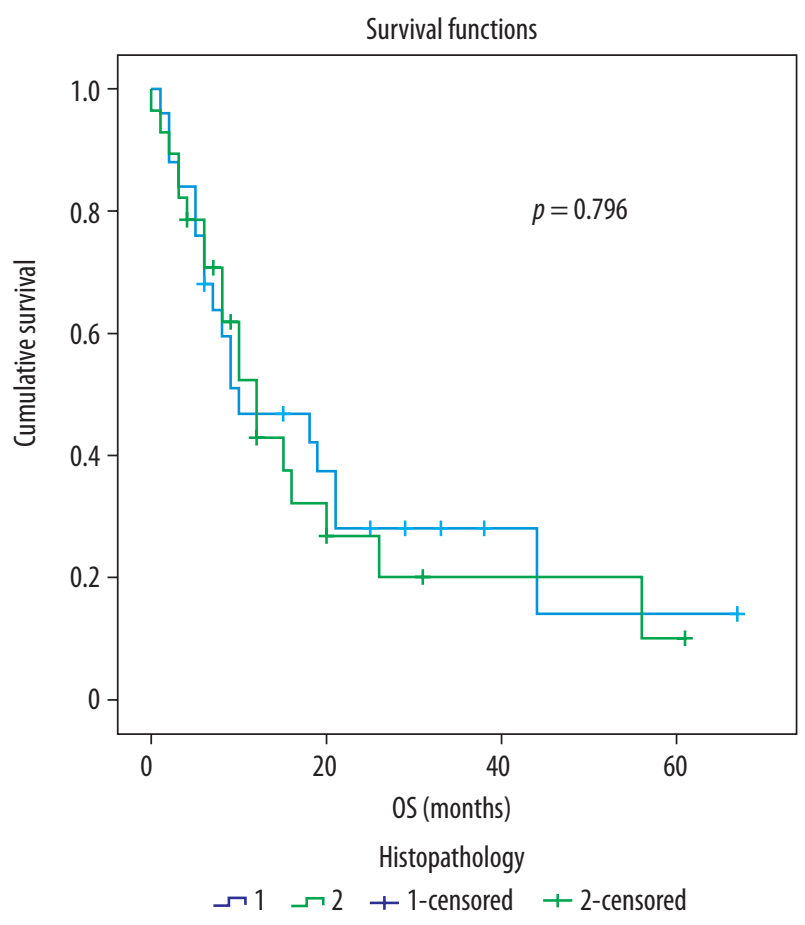

Figure 3. ROC curve analysis of survival rates of the two groups

\section{Metastatic state}

There were no metastases (lymph nodes or organ) in $17(29.8 \%)$ patients. Twenty (35\%) patients had only lymph node metastasis. Distant metastasis was detected in $20(35.1 \%)$ patients. Fifteen $(26.3 \%)$ patients had both organ metastasis and lymph node metastasis. Five patients (8.8\%) had only organ metastasis. The difference between the histological subgroups in terms of metastasis rate was not statistically significant.

The most common sites of metastasis were lungs $27 \%$, adrenal gland $21 \%$, bone $18.9 \%$, and liver $10.8 \%$, respectively. The distribution of metastases according to histological subgroups and organs is given in Table 2.

\section{The relation of metabolic parameters with study subjects}

All patients had FDG-avid tumours at PET/CT.

Survival aspect: The median overall survival of the whole cohort, OACs, and OSCCs was 10 months, 10 months, and 12 months, respectively. There was no difference between

Table 3. Statistical presentation of survival rates of the two groups

\begin{tabular}{|c|c|c|c|c|c|c|c|c|}
\hline \multirow[t]{3}{*}{ Histology } & \multicolumn{4}{|c|}{ Mean } & \multicolumn{4}{|c|}{ Median } \\
\hline & \multirow[t]{2}{*}{ Estimate } & \multirow{2}{*}{$\begin{array}{l}\text { Standard } \\
\text { error }\end{array}$} & \multicolumn{2}{|c|}{$95 \%$ Confidence interval } & \multirow[t]{2}{*}{ Estimate } & \multirow{2}{*}{$\begin{array}{c}\text { Standard } \\
\text { error }\end{array}$} & \multicolumn{2}{|c|}{$95 \%$ Confidence interval } \\
\hline & & & Lower bound & Upper bound & & & Lower bound & Upper bound \\
\hline$O A C$ & 22.289 & 5.004 & 12.480 & 32.097 & 10.000 & 5.831 & .000 & 21.428 \\
\hline oscc & 19.955 & 4.488 & 11.159 & 28.751 & 12.000 & 2.175 & 7.737 & 16.263 \\
\hline Overall & 21.627 & 3.473 & 14.821 & 28.434 & 10.000 & 3.169 & 3.789 & 16.211 \\
\hline
\end{tabular}

$O A C$ - oesophageal adenocancer, $\mathrm{OSCC}$ - oesophageal squamous cell cancer. 
the overall (OS) and progression-free (PFS) survival rates of the two subgroups (statistical data presented in Figure 3 and Table 3).

$\mathrm{SUV}_{\text {max }}$ and TLG aspect: Mean tumour $\mathrm{SUV}_{\text {max }}$ was 11.8 \pm 4.4 for the OAC group, $13.9 \pm 5.4$ for OSCC group, and $12.9 \pm 5$ for the whole cohort $(p=0.193)$. Mean tumour TLG was $316.3 \pm 252.5$ for the OAC group, $283.4 \pm 376$ for the OSCC group, and $299.9 \pm 318$ for the whole cohort $(p=0.249)$. As expected, mean $\mathrm{SUV}_{\max }$ of the primary tumour was higher than those of lymph node and distant metastasis (12.8 vs. 7.2). There was no significant difference between the two histological subgroups for TLG and SUV ${ }_{\text {max }}$.

MTV aspect: MTV was significantly higher in the OAC group. Mean tumour MTV was $30.5 \pm 20.2$ for the OAC group, $21.7 \pm 22.1$ for the OSCC group, and $26 \pm$ 21.4 for the whole cohort $(p=0.017)$. Mean total MTV was $102.9 \pm 159.2$ for the OAC group, $46.31 \pm 67.88$ for the OSCC group, and $74.6 \pm 124.5$ for the whole cohort $(p=0.028)$. Sensitivity, specificity, positive predictive value (PPV), negative predictive value (NPV), and accuracy of metabolic PET parameters are listed as 57.7\%, 84.6\%, 78.9\%, $66.7 \%$, and $71.2 \%$, respectively, for tumour MTV; $76.9 \%$, $61.5 \%, 66.7 \%, 72.7 \%$, and $69.2 \%$, respectively, for total MTV (Figure 4, Table 4).

There was no significant association with MTV, TLG, and $\mathrm{SUV}_{\max }$ for OS or PFS.

Pre-test clinical staging vs. PET/CT staging: According to clinical data before PET/CT imaging, $19 \%$ of the patients were in the early stage, $52.6 \%$ were in the local advanced stage, and $28.1 \%$ were in the metastatic stage. According to the data after PET/CT, 29.8\% of the patients were in the early stage, $35.1 \%$ were in the local advanced stage, and $35.1 \%$ were in the metastatic stage (Table 5).

\section{Discussion}

Although the first and most commonly used PET parameter is $\mathrm{SUV}_{\text {max }}$, an increasing number of studies highlight the prognostic value of other metabolic parameters such as MTV and TLG for oesophageal carcinomas [8,9]. In our study, we found that only the MTV of the primary tumour and whole body was associated the histological subtype and MTV values of OAC significantly higher than OSCC (cut-off value $>26.89$ for both primary tumour MTV and total MTV; detailed sensitivity, specificity, PPV, NPV, and accuracy data are given in Table 4 and Figure 3). A similar cut-off value was suggested by two

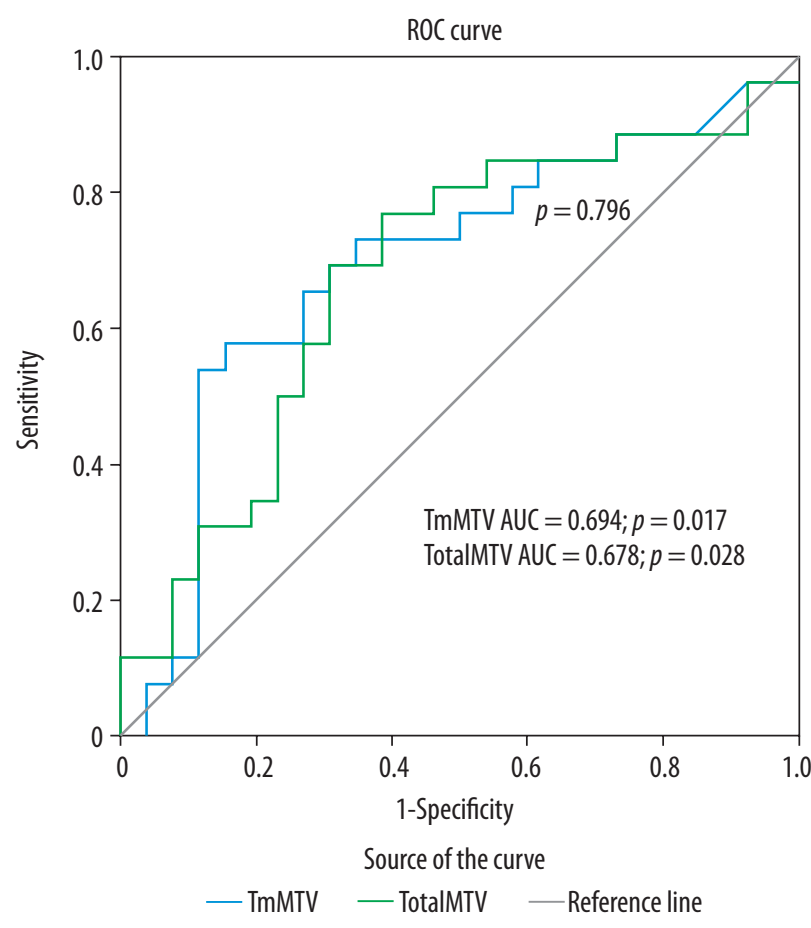

Figure 4. ROC curve analysis of tumour metabolic tumour volume (MTV) and total MTV cut-off values for subgroup prediction

previous studies for the prediction of local tumour recurrence $[10,11]$.

Both TLG and MTV have previously been defined as effective biomarkers for predicting survival and response to treatment in oncological patients $[5,12]$. Fan et al. reported that local recurrence in oesophageal cancer appears mostly within high-FDG-uptake regions. Patients with MTV $\geq 27 \mathrm{~cm}^{3}$ are more inclined to have recurrence in these regions [10]. Similarly, Kim et al. assessed independent risk factors associated with local recurrence and lymphatic metastasis in OC. The results of this study proved that pretreatment MTV is an independent risk factor for local recurrence and lymphatic metastasis. Also, values over $27 \mathrm{~cm}^{3}$ predict recurrence in these metabolically highly active regions. In the same study, TLG has been found as an independent risk factor for lymphatic metastasis [11]. In addition to these studies, which have similar results to ours in terms of MTV cut-off values, we have shown that OAC and OSCC can be distinguished by an MTV value of $>26.89$, and MTV is higher in EACs that tend to have worse prognosis. In our study, $25 \%$ of patients with distant metastasis were OSCC and $44.8 \%$ were OAC, but the difference was not statistically significant. However,

Table 4. Tumour metabolic tumour volume (MTV) and total MTV cut-off values for subgroup prediction

\begin{tabular}{|l|c|c|c|c|c|c|c|c|}
\hline Parameter & Cut-off & AUC & $p$ & $\begin{array}{c}\text { Sensitivity } \\
(\%)\end{array}$ & $\begin{array}{c}\text { Specificity } \\
(\%)\end{array}$ & $\begin{array}{c}\text { PPV } \\
(\%)\end{array}$ & $\begin{array}{c}\text { NPV } \\
(\%)\end{array}$ & $\begin{array}{c}\text { Accuracy } \\
(\%)\end{array}$ \\
\hline TmMTV & $>26.89$ & 0.694 & 0.017 & 57.7 & 84.6 & 78.9 & 66.7 & 71.2 \\
\hline TotalMTV & $>26.89$ & 0.678 & 0.028 & 76.9 & 61.5 & 66.7 & 72.7 & 69.2 \\
\hline
\end{tabular}

NS: $p>0.05$, TmMTV - primary tumour's metabolic tumour volume, TotalMTV - total metabolic tumour volume 
Table 5. Comparison of pre-test clinical staging and positron emission tomography (PET) combined with computed tomography (CT) staging results of the cohort

\begin{tabular}{|c|c|c|}
\hline Staging method & $n$ & $\%$ \\
\hline \multicolumn{3}{|l|}{ Clinical } \\
\hline Early stage & 11 & 19.3 \\
\hline Locally advanced stage & 30 & 52.6 \\
\hline Metastatic stage & 16 & 28.1 \\
\hline \multicolumn{3}{|l|}{$\mathrm{PET} / \mathrm{CT}$} \\
\hline Early stage & 17 & 29.8 \\
\hline Locally advanced stage & 20 & 35.1 \\
\hline Metastatic stage & 20 & 35.1 \\
\hline
\end{tabular}

OACs appear to be more willing to metastasise. After PET/ CT imaging, nine (15.8\%) patients (six OAC and three OSCC) were upstaged and 12 (21\%) patients (five OAC and seven OSCC) were downstaged. Overall, the disease stage changed in 20 (36.9\%) of 50 patients.

MTV has been suggested as one of the important independent prognostic factors, predicting survival better than $\mathrm{SUV}_{\max }$ for primary tumours in patients with OC [13]. Our results showed that MTV is the only parameter that differs from the histological subtype. Although our results did not show significant differences between histological subgroups in terms of OS and PFS, this change in MTV values could be used to predict the progression of the disease.

There is no standard cut-off value for metabolic response to treatment. PERCIST recommends a $30 \%$ reduction in $\mathrm{SUV}_{\text {max }}$ as a cut-off value in solid tumours, but there is no defined value for MTV [14]. Although, some studies assert that SUV values are limited in predicting histological CRT response and pathological complete remission in OC, the same studies also reveal that MTV may be a better predictor of response [4]. Recently, Zhang et al. investigated the prognostic value of interim FDG PET in order to determine the objective tumour response for CRT in 47 patients with OSCC, and they reported that MTV tended to be decreased from baseline to interim PET. Based on these data, a threshold of $54 \%$ alteration on MTV distinguishes responders to therapy from those who do not respond [5]. Soydal et al. showed that patients who have higher preoperative MTVs could be good candidates for more aggressive CRT regiments [15].

Likewise, in another study by Li et al., in 2015, initial evaluation of MTV was observed to be an independent factor for OS in patients with OSCC, who have received chemotherapy alone. In the same study, optimal initial MTV and TLG thresholds for predicting overall survival was determined as $22 \mathrm{~cm}^{3}$ and 170, respectively. In this regard, it was interpreted that patients with lower MTV and TLG levels may have longer survival time [16]. Although the distribution of histologic subgroups in our cohort was balanced, we could not observe the predictive value of MTV in terms of response to treatment, which may be due to the limited number of patients.

Chhabra et al. investigated the predictive values of SU$\mathrm{V}_{\max }$ and MTV for survival among 57 patients who were diagnosed with OSCC. Their results affirm that the baseline $\mathrm{SUV}_{\text {max }}$ and MTV are significantly associated with better OS. Interestingly, detailed analysis of post-CRT data shows that MTV is the only prognostic parameter for OS in univariate analysis while SUVmax is prognostic for DFS in multivariate analysis [17]. The findings of Schuurbiers et al. show that OSSC generally has higher FDG uptake, which is considered as a poor prognostic factor, but OACs have worse DFS and greater potential for metastasis, with an explanation that squamous cell carcinomas exhibit glycolysis essentially under hypoxic conditions [18].

Certain studies have demonstrated that $\mathrm{SUV}_{\text {max }}$ is an independent predictor of stage and a determinant of survival in patients with OC $[8,19]$. However, in our study, we did not observe a relationship between histological subtypes in terms of TLG and $\mathrm{SUV}_{\text {max }}$.

The major limitations of our study were the low number of cases, the retrospective design of the study, and the heterogeneity of the treatment protocols of patients, which is due to the clinical variation and the individuality of the treatment. We believe that the inclusion of the histological subgroups brought a fresh, although limited, perspective to the prognostic value of MTV and TLG.

\section{Conclusions}

This study assessed whether PET/CT defined MTV, TLG, and $\mathrm{SUV}_{\max }$ would differentiate patient endpoints (live vs. dead), and whether they could discriminate between the two histological subtypes (OAC vs. OSCC) of OC. Our results were revealed in the interpretation of the question. MTV has the ability to distinguish between histological subgroups of OC with $>26.9$ cut-off value. The pre-treatment MTV value can be helpful in evaluating the prognosis.

\section{Conflict of interest}

The authors report no conflict of interest.

\section{References}

1. Bray F, Ferlay J, Soerjomataram I, Siegel RL, Torre LA, Jemal A. Global cancer statistics 2018: GLOBOCAN estimates of incidence and mortality worldwide for 36 cancers in 185 countries. CA: A Cancer J Clin 2018; 68: 394-424. 
2. Arnold M, Soerjomataram I, Ferlay J, Forman D. Global incidence of oesophageal cancer by histological subtype in 2012. Gut 2015; 64: 381-387.

3. Stahl M, Stuschke M, Lehmann N, et al. Chemoradiation with and without surgery in patients with locally advanced squamous cell carcinoma of the esophagus. J Clin Oncol 2005; 23: 2310-2317.

4. Harada K, Kaya DM, Lopez A, et al. Personalized therapy based on image for esophageal or gastroesophageal junction adenocarcinoma. Ann Transl Med 2018; 6: 80.

5. Zhang P, Li Z, Wang D, et al. 18F-fluorodeoxyglucose positron emission computed tomography for monitoring tumor response in esophageal carcinoma treated with concurrent chemoradiotherapy. Oncol Lett 2018; 15: 1845-1852.

6. Van De Wiele C, Kruse V, Smeets P, et al. Predictive and prognostic value of metabolic tumour volume and total lesion glycolysis in solid tumours. Eur J Nucl Med Mol Imaging 2013; 40: 290-301.

7. Usmanij EA, De Geus-Oei LF, Troost EGC, et al. 18F-FDG PET early response evaluation of locally advanced non-small cell lung cancer treated withconcomitant chemoradiotherapy. J Nucl Med 2013; 54: 1528-1534.

8. Sahiner I, Atasever T, Akdemir UO, et al. Relationship between primary lesion metabolic parameters and clinical stagein lung cancer. Rev Esp Med Nucl Imagen Mol 2013; 32: 357-363.

9. Ho Seok I, Kim SJ, Kim IJ, Kim K. Predictive value of metabolic tumor volume measured by $18 \mathrm{~F}-\mathrm{FDG}$ PET for regional lymph node status in patients with esophageal cancer. Clin Nucl Med 2012; 37: 442-446.

10. Fan B, Fan P, Kong L, et al. 18F-deoxyglucose positron emission tomography/computed tomography to predict local failure in esophageal squamous cell carcinoma. Oncotarget 2017; 8: 34498-34506.

11. Kim SJ, Pak K, Chang S. Determination of regional lymph node status using 18F-FDG PET/CT parameters in oesophageal cancer patients: comparison of SUV, volumetric parameters and intratumoral heterogeneity. Br J Radiol 2016; 89: 20150673.

12. Yildirim BA, Torun N, Guler OC, Onal C. Prognostic value of metabolic tumor volume and total lesion glycolysis in esophageal carcinoma patients treated with definitive chemoradiotherapy. Nucl Med Commun 2018; 39: 553-563.

13. Hyun SH, Choi JY, Shim YM, et al. Prognostic value of metabolic tumor volume measured by $18 \mathrm{~F}$-fluorodeoxyglucose positron emission tomography in patients with esophageal carcinoma. Ann Surg Oncol 2010; 17: 115-122.

14. Wahl RL, Jacene H, Kasamon Y, Lodge MA. From RECIST to PERCIST: evolving considerations for PET response criteria in solid tumors. J Nucl Med 2009; 50 Suppl 1: 122S-150S.

15. Soydal C, Yüksel C, Küçük ON, et al. Prognostic value of metabolic tumor volume measured by 18F-FDG PET/CT in esophageal cancer patients. Mol Imaging Radionucl Ther 2014; 23: 12-15.

16. Li Y, Lin Q, Luo Z, et al. Value of sequential 18F-fluorodeoxyglucose positron emission tomography/computed tomography (FDG PET/ CT) in prediction of the overall survival of esophageal cancer patients treated with chemoradiotherapy. Int J Clin Exp Med 2015; 8: 1094710955.

17. Chhabra A, Ong LT, Kuk D, et al. Prognostic significance of PET assessment of metabolic response to therapy in oesophageal squamous cell carcinoma. Br J Cancer 2015; 113: 1658-1665.

18. Schuurbiers OC, Meijer TW, Kaanders JH, et al. Glucose metabolism in NSCLC is histology-specific and diverges the prognostic potential of 18 FDG-PET for adenocarcinoma and squamous cell carcinoma. J Thorac Oncol 2014; 9: 1485-1493.

19. Cerfolio RJ, Bryant AS. Maximum standardized uptake values on positron emission tomography of esophageal cancer predicts stage, tumor biology, and survival. Ann Thorac Surg 2006; 82: 391-395. 